Classification

Physics Abstracts

$81.60 \mathrm{~J}-82.65 \mathrm{D}$

\title{
Wetting of polymer covered surfaces
}

\author{
A. Halperin and P. G. de Gennes \\ Collège de France, Place Marcelin-Berthelot, 75231 Paris Cedex 05, France
}

(Reçu le 28 janvier 1986, accepté le 20 mars 1986)

\begin{abstract}
Résumé. - Nous discutons le comportement de surfaces greffées, ou couvertes de polymères adsorbés, et exposées à un liquide qui est un bon solvant du polymère. Notre premier objectif est le paramètre d'étalement $S$, que nous calculons en fonction de la longueur des chaînes, de la qualité du solvant, et aussi de la force de l'adsorption. Par ailleurs, nous analysons les films de mouillage, obtenus à $S>0$ par étalement d'un solvant non volatil. L'épaisseur $e$ du film est un compromis entre $S$ (qui favorise des films minces) et l'entropie des chaînes confinées dans le film (qui favorise des films épais). Dans la plupart des cas, l'épaisseur d'équilibre $e$ s'avère plutôt petite. Enfin, nous analysons l'étalement sur une surface bigarrée : par exemple, une zone circulaire couverte de polymère, entourée par du solide nu, ou l'inverse. Dans ces situations, l'épaisseur du film sur la zone centrale doit changer brutalement quand la goutte atteint le bord de cette zone.
\end{abstract}

\begin{abstract}
We consider solid surfaces, partly covered with flexible, neutral, linear polymers (by adsorption or by grafting), wetted by a liquid which is a good solvent of the poymer. We give formulae for the spreading coefficient $S$ as a function of chain length, solvent quality and adsorption strength. We also discuss the wetting films obtained in spreading a droplet of (non volatile) solvent : the equilibrium thickness $e$ of the film is a compromise between $S$ (favouring thin films) and the coil entropies (favouring thick films) : in most cases $e$ is rather small. Finally we analyse the spreading on certain "patchy " coatings where the film thicknesses can change dramatically with time.
\end{abstract}

\section{Introduction.}

The spreading of a liquid on a surface may be affected by the presence of polymers. Such effects may be of importance to certain practical applications like adhesion, detergency, etc. Because of the large scale imposed by the polymers it is convenient to investigate these systems using a continuum theory of wetting [1]. The spreading of dilute [2] and of semi dilute [3] polymer solutions on a non adsorbing surface were recently analysed within this framework. Here we consider the spreading of a pure non volatile solvent on a polymer coated solid. We distinguish between surfaces which are coated by grafted polymers and those coated by adsorbed polymers.

1) The tendency for a fluid to wet a solid surface is determined by the spreading coefficient [1] $S$

$$
S=f_{\mathrm{d}}-f_{\mathrm{w}}
$$

where $f_{\mathrm{d}}$ and $f_{\mathrm{w}}$ are respectively the free energies per unit area of the dry and the wet surface. It should be stressed that $f_{\mathrm{w}}$ refers to a surface covered by a macroscopic liquid layer. We derive explicit formulae for $S$ in section 1 for a grafted polymers, and in section 3 for adsorbed polymers.

2) We also discuss spreading : i.e. a non volatile liquid invading a dry solid surface with $S>0$. In this case a drop of liquid transforms into a flat « pancake ». The equilibrium thickness of the pancake (e) is determined by the balance between $S$ and long range forces which tend to thicken the film. Neglecting edge effects the free energy of the film is

$$
f=f_{0}+(P(e)-S) A
$$

where $P(e)$ denotes long range interactions and is such that $P(e \rightarrow \infty)=0$. $A$ is the total area of the film. For our purposes $P$ consists of polymer comtributions to be discussed below plus non retarded Van der Waals interactions given by

$$
P_{\mathrm{vw}}(Z)=\frac{A_{\mathrm{H}}}{12 \pi} \frac{1}{e^{2}}
$$

where $A_{\mathrm{H}}$ is a Hamaker constant. To obtain the equilibrium thickness we minimize the free energy 
with respect to $e$ at constant volume $V=A e$ which yields [4]

$$
S=e \Pi(e)+P(e)
$$

where $\Pi(e)$, the disjoining pressure, is defined by $\Pi=-\mathrm{d} P / \mathrm{d} e$.

\section{Wetting of a grafted surface.}

2.1 SPREAdING COEFFICIENT. - Here we discuss the wetting of a uniform, non adsorptive, surface on which linear, flexible, uncharged, polymers with $N$ monomers/chain are grafted. We denote the average distance between grafted sites by $D$, and consider the "dense " case $D \ll R_{\mathrm{F}}$ where $R_{\mathrm{F}}$ is the size of a free coil. The graft density is given by

$$
\sigma=\left(\frac{a}{D}\right)^{2}<1
$$

where $a$ is the mesh size of the appropriate FloryHuggins lattice. When the surface is covered by a macroscopic layer of a good solvent the chains are stretched, and the thickness of the grafted layer is $[5,6]$

$$
L \simeq N a \sigma^{1 / 3} .
$$

The concentration profile in this case is essentially flat with monomer volume fraction

$$
\phi \simeq \sigma^{2 / 3} \ll 1 .
$$

As the grafted layer is mostly uniform and rather dense the Flory mixing free energy provides an appropriate description of it, once we discard the translational term, which is suppresed by the grafting. The free energy per lattice site is then

$$
\begin{aligned}
\frac{F_{\text {mix }}}{k T} & =(1-\phi) \ln (1-\phi)+X \phi(1-\phi) \\
& \simeq \phi(-1+X)+\frac{1}{2} \phi^{2}(1-2 X) \phi \ll 1 .
\end{aligned}
$$

To obtain $S$ we now write [7]

$$
\begin{aligned}
& f_{\mathrm{d}}=f_{\mathrm{d}}^{\text {bulk }}+\gamma_{\mathrm{pv}}+\gamma_{\mathrm{sp}}(1-\sigma) \\
& f_{\mathrm{w}}=f_{\mathrm{d}}^{\text {bulk }}+\Gamma F_{\text {mix }}+\gamma+\gamma_{\mathrm{sl}}(1-\sigma)
\end{aligned}
$$

where $\gamma_{\mathrm{sp}}, \gamma_{\mathrm{pv}}, \gamma$, and $\gamma_{\mathrm{sl}}$ are respectively the solidpolymer, polymer-vapour, liquid-vapour and solidliquid surface tensions. $\Gamma$, the total number of monomers per unit area is given by $\Gamma=N / D^{2}$. The factors $(1-\sigma)$ in $(2.5)$ describe the reduction is solid-fluid contacts due to the grafted sites. Using equation (1.1) we find

$$
\begin{aligned}
& S=S_{0}+k T \Gamma\left[(1-X)-\frac{1}{2} \phi(1-2 X)+\cdots\right] \\
& S_{0}=\gamma_{\mathrm{pv}}-\gamma+\left(\gamma_{\mathrm{sp}}-\gamma_{\mathrm{sl}}\right)(1-\sigma) .
\end{aligned}
$$

It should be noted that $k T \Gamma$ is rather large. For $N=10^{2}$ and $D=10 \AA, k T \Gamma \simeq 5 \times 10^{2} \mathrm{erg} / \mathrm{cm}^{2}$. Thus, in many cases we may have $S \simeq k T \Gamma$, i.e. the spreading is driven mainly by entropy of dissolution.

2.2 STRUCtURe OF A WeTting FiLM. - Because $S \simeq k T \Gamma$ is very large, the wetting film ruled by equation (1.4) tends to be very thin. Accordingly, the polymer volume fraction may approach unity. A rough form of $\Pi$ in this regime may be obtained by using a Flory type argument. We start by writing the free energy of a single chain as a sum of entropic and elastic terms [6]

$$
\frac{1}{k T} F=\frac{e D^{2}}{a^{3}}(1-\phi) \ln (1-\phi)+\frac{3 e^{2}}{2 R_{0}^{2}} .
$$

Here we identify the thickness of the wetting layer with that of the grafted layer, $e D^{2} / a^{3}$ is the number of lattice sites per grafted chain and $R_{0}=N^{1 / 2} a$. To obtain $\Pi$ we use

$$
\Pi=-\frac{\partial F}{\partial e}
$$

keeping in mind the constraint

$$
\phi=\frac{N a^{3}}{e D^{2}}=N \sigma \frac{a}{e} .
$$

The resulting expression for $\Pi$ is

$$
\begin{aligned}
\frac{\Pi}{k T} \simeq \frac{1}{a^{3}}[-\ln (1- & \left.N \sigma \frac{a}{e}\right)-N \sigma \frac{a}{e}- \\
& \left.-2 \frac{\sigma}{N} \frac{e}{a}+2 N \sigma\left(\frac{a}{e}\right)^{3}\right]
\end{aligned}
$$

Note that for small $e$ values, $\Pi$ is dominated by the entropic term. The corresponding $P$ function is then

$$
\begin{aligned}
\frac{P(e)}{k T} & \simeq \frac{e}{a^{3}}\left[\left(1-N \sigma \frac{a}{e}\right) \ln \left(1-N \sigma \frac{a}{e}\right)+\right. \\
+ & \left.+\frac{\sigma}{N} \frac{e}{a}+N \sigma\left(\frac{a}{e}\right)^{3}\right]+\cdots+\text { constants of order } \sigma
\end{aligned}
$$

When $S$ is dominated by the polymer $(S \simeq k T \Gamma)$, (1.4), (2.10) and (2.11) lead to

$$
\begin{aligned}
&-\ln (1-\phi)-\phi- \sigma^{2} \frac{1}{\sigma}+3 \frac{1}{(N \sigma)^{2}} \phi^{3}+ \\
&+(1-\phi) \ln (1-\phi) \simeq \phi
\end{aligned}
$$

which is solved by

$$
\begin{aligned}
& \phi \simeq 1-\exp (-2) \sim 0.9 \\
& e \sim N \sigma a .
\end{aligned}
$$


Note that in comparison with a grafted layer immersed in a solvent bath, the present layer is predicted to be highly compressed $\left(e \ll N \sigma^{1 / 3} a\right)$.

\section{Wetting of a surface coated by adsorbed polymer.}

In this section we consider the wetting of a uniform solid surface coated by adsorbed polymer molecules. The structure is a diffuse self-similar layer; in most parts of the layer the concentration is low, thus justifing the use of available scaling results [8-10]. Our analysis depends on a reproducible behaviour of the adsorbed polymers following wetting-drying cycles, i.e. we assume that the concentration profile obtained by wetting a dry surface carrying adsorbed polymers, is identical to the one obtained by incubating the bare surface in a polymer solution. It is natural to assume that, on the dry surface, the polymer builds up a completely dense layer. On the basis of those assumptions we can write

$$
\begin{aligned}
& f_{\mathrm{d}}=\phi_{\mathrm{ds}}\left(\gamma_{\mathrm{sp}}+\gamma_{\mathrm{pv}}\right)+\left(1-\phi_{\mathrm{ds}}\right) \gamma_{\mathrm{sv}} \\
& f_{\mathrm{w}}=\gamma_{\mathrm{sl}}+\gamma+\Delta \gamma
\end{aligned}
$$

$\phi_{\mathrm{ds}}$ denotes the fraction of the dry surface which is covered by monomers. In this case

$$
\phi_{\mathrm{ds}}= \begin{cases}\Gamma a^{2} & \Gamma a^{2}<1 \\ 1 & \Gamma a^{2} \geqslant 1\end{cases}
$$

Let us first define the strength of the attraction in terms of a dimensionless parameter $\delta(-k T \delta$ is the attractive energy felt by a monomer in contact with the solid surface). Most practical situations correspond to $\delta \sim 1$. However, the regime of small $\delta$ can also be understood using data on a certain "crossover exponent " [10-12] and is interesting : thus we shall first consider the limit of $\delta$ small. The essential feature is the existence of a characteristic length $D(\delta)$ : at distances $z>D(\delta)$ from the wall, the adsorbed layer has the standard ( $\delta$ independent) self-similar profile $\phi \sim z^{-4 / 3}$, while at distances $z<D(\delta)$ a different «proximal» regime prevails. It turns out numerically that $D(\delta) \sim|\delta|^{-\kappa} a$ with $\kappa=1$ to a good approximation [11].

Knowing the structures of $D(\delta)$ we can immediately construct the scaling structure of the surface energy correction $\Delta \gamma$. In a strong depletion regime $(\delta<0$, $\delta \sim-1)$ we have [13]

$$
\Delta \gamma \sim k T / \xi_{\mathrm{b}}^{2}
$$

In a general case ( $\delta$ small, but of arbitrary sign) we then expect

$$
\Delta \gamma=\frac{k T}{\xi_{\mathrm{b}}^{2}} f\left(\frac{\xi_{\mathrm{b}}}{D(\delta)}\right)
$$

where $f$ is a dimensionless function. In the particular regime of interest here $\left(\delta>0 ; \phi_{\mathrm{b}} \rightarrow 0, \quad \xi_{\mathrm{b}} \rightarrow \infty\right)$ we must have an $\Delta \gamma$ which is finite, and thus independent of $\xi_{\mathrm{b}}$. This imposes $f(x \rightarrow \infty) \rightarrow x^{2}$ and gives

$$
\Delta \gamma=\frac{k T}{D^{2}(\delta)}=\frac{k T}{a^{2}} \delta^{2}
$$

combining (1.1), (3.1) and (3.5) we find

$$
\begin{aligned}
& S \simeq S_{0}+\frac{k T}{D^{2}} \\
& S_{0}=\Gamma a^{2}\left(\gamma_{\mathrm{sp}}+\gamma_{\mathrm{pv}}-\gamma_{\mathrm{sv}}\right)+\gamma_{\mathrm{sv}}-\gamma_{\mathrm{sl}}-\gamma .
\end{aligned}
$$

For strong adsorption i.e. $\delta \sim 1, D(\delta) \sim a$ and the situation is similar to that at the grafted surface. $S$ is dominated by the $k T / a^{2}$ term and the wetting film will tend to be very thin. In this case, though, the thickness of the film will be at order $a$, and the use at a continuum theory becomes questionable. No such difficulties are encountered in the case at weak adsorption i.e. $\delta \ll 1$, but the situation is then less interesting because $S \simeq S_{0}$. In the special case of wetting by a liquid monomer we may expect the correction term $k T / D^{2}(\delta)$ to be the dominant one. Because $k T / D^{2}(\delta)$ is comparatively small we expect the wetting film to be rather thick. We may then identify $\Pi$ with the force between two plates coated by adsorbed polymer in the self-similar regime [9]

$$
\Pi \sim \frac{k T}{e^{3}} .
$$

We then find, using (1.4), a film thickness :

$$
e \sim D(\delta) \text {. }
$$

\section{Wetting of a patchy surface.}

In preceding sections we have considered the wetting of uniform surfaces. Now we turn to patchy surfaces; we discuss two geometries (Fig. 1) : a circular patch coated with polymers and surrounded by the bare surface, and the inverse geometry, a bare circular

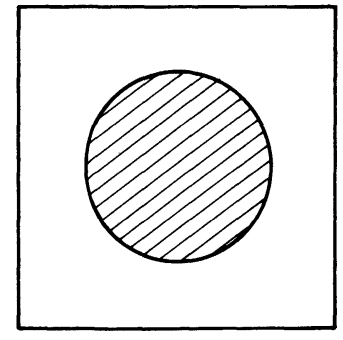

(a)

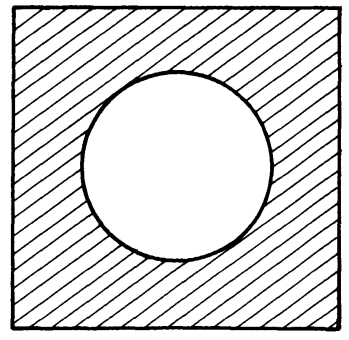

(b)
Fig. 1. - The two coating geometries considered for the wetting of a patchy surface. Hatched areas are coated by polymers. 
patch surrounded by a coated surface. We imagine that the centre of the structure is fed by a pipette, building up an adiabatically growing pancake, whose centre coincides with that of the patch. (1) When the wetting film is confined to the patch it is possible to analyse it by using the method outlined in previous sections (2) once the film has spread past the patch boundaries we expect the film to have two zones which differ in their thickness. Furthermore, we will see that the thickness of the film at the central zone (on the patch) undergoes an abrupt change once the contact line reaches the peripheral zone. Equation (1.4) describes the balance of forces at the contact line. Thus it applies only to the peripheral zone which is delineated by the contact line and the patch boundary. To obtain the equilibrium thickness of the central zone we invoke the equality of the solvent chemical potential in the two zones. In this case this equality implies (see appendix)

$$
\Pi_{\mathrm{c}}\left(e_{\mathrm{c}}\right)=\Pi_{\mathrm{p}}\left(e_{\mathrm{p}}\right)
$$

where $\Pi$ is always the disjoining pressure, $e_{\mathrm{c}}$ and $e_{\mathrm{p}}$ are respectively the equilibrium thickness of the central and peripheral zones.

The equilibrium thickness of the film at the central zone is determined by the $S$ felt by the film at the peripheral zone. The nature of the "patch " surface determines the functional form of $\Pi$ in the central zone.

Let us now consider the same two geometries when the surface is coated by weakly adsorbed polymers. When the outer zone is coated, the thickness of the peripheral film is given by (3.8). Thus, equations (4.1) and (3.7) lead to

$$
\epsilon_{\mathrm{c}}=\left(\frac{\gamma a^{2}}{k T}\right)^{1 / 3} D
$$

where we have taken $A_{\mathrm{H}}$ to be given by [1]

$$
A_{\mathrm{H}}=6 \pi \gamma a \text {. }
$$

In the opposite case where the central zone is coated, the thickness of the film at the peripheral zone is known [1] to be

$$
e_{\mathrm{p}}=a\left(\frac{3 \gamma}{2 S}\right)^{1 / 2}
$$

and thus equation (4.1) leads to

$$
e_{\mathrm{c}}=a\left(\frac{k T}{\gamma a^{2}}\right)^{1 / 3}\left(\frac{3 \gamma}{2 S}\right)^{1 / 2} .
$$

In both cases the film thickness at the central zone changes abruptly once the contact line overtakes the patch boundary (Fig. 2). This effect is more pronounced when we consider grafted surfaces. In the case of a bare central zone, $e_{\mathrm{c}}$ changes from the value given in (4.4) to $\sim a$. (As a continuum theory is not valid on this scale this result must not be taken

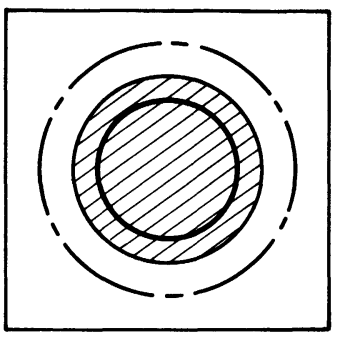

Fig. 2. - Dry spreading on a patchy surface. The film thickness in the central (hatched) zone changes abruptly when the contact line overtakes the patch boundary. - A contact line inside the patch boundary. - . - A contact line outside the patch boundary.

literally, but only as an indication of a trend.) When the central zone is grafted and the peripheral surface is bare, $e_{\mathrm{c}}$ is given, in the limit of $S / \gamma \ll 1$, by

$$
e_{\mathrm{c}} \simeq\left(\frac{3}{2} \frac{\gamma}{S}\right)^{3 / 4} N \sigma a
$$

Thus in this case $e_{\mathrm{c}}$ is significantly larger than $N \sigma a$, its thickness before the contact line reached the bare surface.

\section{Conclusions.}

1) Grafting or adsorbing polymer on a solid surface leads to a dramatic change in it's wettability : this is no surprise, but our equations show quantitatively what is the role of the various control parameters : polymer length, grafting density, solvent quality and adsorption strength.

2) Where we spread a good, (non volatile) solvent on a polymer coated surface the spreading coefficient $S$ is so large that the final wetting film is usually very thin : this regime cannot be analysed very well by our continuum theory but the qualitative trends are clear.

3) If the surface coating is patchy, we can find some remarkable scenarios of spreading, with films which transit from a thick state to a thin state, etc. Patchy surfaces will often be present in practice; they lead to classical hysteresis effects in partial wetting. But we see here that they can also give remarkable features in complete wetting.

Our discussion was limited to simple, circular or annular patches. With disordered patches, certain interesting statistical effects come into play [14, 15].

\section{Acknowledgments.}

We have greatly benefited from discussions with M. Boudoussier, F. Brochard, J. F. Joanny and L. Leibler. 
Appendix.

Chemical potential of the solvent. - Equation (4 . 1) expresses the equality of the solvent chemical potentials $\mu_{\mathrm{s}}$ in the two films. We rederive below the relation between $\mu_{\mathrm{s}}$ and the disjoining pressure $\Pi$, for the specific case of a film in dry spreading.

Consider a film of area $A$, thickness $e$, and vary both $A$ and $e$. The resulting change in free energy from equation (1.2) is

$$
\mathrm{d} F=\mathrm{d} A(P-S)-\Pi \mathrm{d} e .
$$

If the film is in equilibrium, this means that $\mathrm{d} F=0$ when the number of solvent molecules $N_{\mathrm{s}}$ is fixed; more generally we must have

$$
\mathrm{d} F=\mu_{\mathrm{s}} \mathrm{d} N_{\mathrm{s}} .
$$

Since the polymer is stuck to the wall, all the change in volume $\mathrm{d}(A e)$ is due to $\mathrm{d} N_{\mathrm{s}}$

$$
a^{3} \mathrm{~d} N_{\mathrm{s}}=A \mathrm{~d} e+e \mathrm{~d} A .
$$

Inserting (A.2, 3) into (A . 1) we get two equations for $\mu$

$$
\mu_{\mathrm{s}}=\frac{a^{3}}{e}(P-S)=-a^{3} \Pi \text {. }
$$

The second form shows the relation between $\mu_{\mathrm{s}}$ and $\Pi$. Also the equality of the two forms leads back to (1.4).

In our patch problem, with two films in equilibrium, the centre film has a fixed $A$. For this film equation (A.1) is reduced to

$$
\mathrm{d} F=-\Pi\left(e_{\mathrm{c}}\right) \mathrm{d} e_{\mathrm{c}}
$$

while for the outer film (variable $A$ and fixed spreading coefficient $(S)$ ) both forms of equation (A.4) hold.

\section{References}

[1] De Gennes, P. G., Rev. Mod. Phys. 57 (1985) 827.

[2] Halperin, A., Pincus, P., AleXander, S., J. Physique Lett. 46 (1985) L-543.

[3] Boudoussier, M., to be published.

[4] Joanny, J. F., De Gennes, P. G., C.R. Heb. Sean. Acad. Sci. 299.II (1984) 279.

[5] Alexander, S., J. Physique 38 (1977) 983.

[6] De Gennes, P. G., Macromolecules 13 (1980) 1075.

[7] De Gennes, P. G., Lecture at the Collège de France (1985).

[8] De Gennes, P. G., Macromolécules 14 (1981) 1637.
[9] De Gennes, P. G., Macromolécules 15 (1982) 492.

[10] De Gennes, P. G., Pincus, P., J. Physique Lett. 44 (1983) L-241.

[11] Eisenriegler, E., Kremer, K., Binder, K., J. Chem. Phys. 77 (1982) 6296.

[12] Ishinabe, T., J. Chem. Phys. 76 (1982) 5589.

[13] Joanny, J. F., Leibler, L., De Gennes, P. G., J. Polym. Sci. Polym. Phys. Ed. 17 (1979) 1073.

[14] De Gennes, P. G., C.R. Heb. Séan. Acad. Sci. 300.II (1985) 129.

[15] JoANNY, J. F., to be published. 